\title{
Lgals3bp suppresses colon inflammation and tumorigenesis through the downregulation of TAK1-NF-KB signaling
}

Sang-Hee Cho ${ }^{1,2}$, Hyun-Jeong Shim', Mi-Ra Park', Ji-Na Choi ${ }^{1}$, Md Rashedunnabi Akanda $\mathbb{1}^{3,4,5}$, Jun-Eul Hwang ${ }^{1}$, Woo-Kyun Bae $e^{1,3}$, Kyung-Hwa Lee $e^{2,3,4}$, Eun-Gene Sun (i) ${ }^{1}$ and Ik-Joo Chung ${ }^{1,2}$

\begin{abstract}
Galectin 3-binding protein (LGALS3BP, also known as 90K) is a multifunctional glycoprotein involved in immunity and cancer. However, its precise role in colon inflammation and tumorigenesis remains unclear. Here, we showed that $\mathrm{Lgals} 3 \mathrm{bp}^{-1-}$ mice were highly susceptible to colitis and colon tumorigenesis, accompanied by the induction of

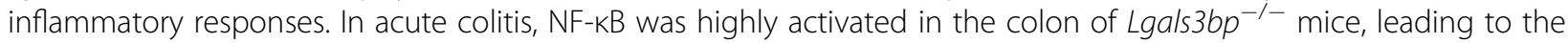
excessive production of pro-inflammatory cytokines, such as IL-6, TNFa, and IL-1 $\beta$. Mechanistically, Lgals3bp suppressed NF-KB through the downregulation of TAK1 in colon epithelial cells. There was no significant difference in the pro-inflammatory cytokine levels between wild-type and $\mathrm{Lgals}_{\mathrm{S}} \mathrm{bp}^{-1-}$ mice in a chronic inflammatory state, during colon tumorigenesis. Instead, Lgals $36 p^{-1-}$ mice showed elevated levels of GM-CSF, compared to those in WT mice. We also found that GM-CSF promoted the accumulation of myeloid-derived suppressor cells and ultimately increased colon tumorigenesis in $\mathrm{Lgals} 3 \mathrm{bp}^{-1-}$ mice. Taken together, Lgals3bp plays a critical role in the suppression of colitis and colon tumorigenesis through the downregulation of the TAK1-NF-KB-Cytokine axis. These findings suggest that LGALS3BP is a novel immunotherapeutic target for colon inflammation and tumorigenesis.
\end{abstract}

\section{Introduction}

Inflammatory bowel disease (IBD) is an inflammatory disorder of the gastrointestinal tract. Excessive, chronic inflammation promotes colorectal cancer (CRC) development. Therefore, IBD patients are at high risk of CRC development ${ }^{1,2}$. CRC is the fourth most lethal malignancy worldwide $^{3}$. Overall, cancer therapies, such as surgery, radiotherapy, and chemotherapy, fail to restrict CRC metastasis ${ }^{4}$. Therefore, it is necessary to further elucidate

\footnotetext{
Correspondence: Eun-Gene Sun (egsun@chonnam.ac.kr) or Ik-

Joo Chung (ijchung@chonnam.ac.kr)

'Department of Hematology and Oncology, Chonnam National University Medical School and Hwasun Hospital, Hwasun, Republic of Korea

${ }^{2}$ Immunotherapy Innovation Center, Chonnam National University Medical School and Hwasun Hospital, Hwasun, Republic of Korea

Full list of author information is available at the end of the article

These authors contributed equally: Sang-Hee Cho, Hyun-Jeong Shim

Edited by Alessandro Rufini
}

the molecular mechanisms underlying IBD pathogenesis and CRC progression.

$\mathrm{NF}-\mathrm{kB}$ is a critical regulator of inflammation, cancer, and immunity ${ }^{5,6}$. Many cellular stimuli activate the NF- $\kappa B$ pathway through transforming growth factor- $\beta$-activated kinase 1 (TAK1) $)^{7}$. TAK1 was originally identified as a transforming growth factor- $\beta$ (TGF- $\beta$ )-activated mitogenactivated protein kinase kinase kinase ${ }^{8}$. However, it was later characterized and widely accepted as a key player in inducing pro-inflammatory cytokine signals, including tumor necrosis factor- $\alpha$ (TNF- $\alpha$ ), interleukin-1 (IL-1), and TLR ligands ${ }^{9}$. Because TNF- $\alpha$ and IL-1 not only stimulate TAK1-NF- $\mathrm{kB}$ signaling but also target genes, positive feedback loops are induced in a paracrine or autocrine manner ${ }^{10}$. Hyper-activated TAK1 signaling causes severe inflammatory disorders and inflammationassociated cancer ${ }^{9}$. Thus, targeting TAK1 is a potential

\section{(c) The Author(s) 2021}

(c) (i) Open Access This article is licensed under a Creative Commons Attribution 4.0 International License, which permits use, sharing, adaptation, distribution and reproduction cc) in any medium or format, as long as you give appropriate credit to the original author(s) and the source, provide a link to the Creative Commons license, and indicate if changes were made. The images or other third party material in this article are included in the article's Creative Commons license, unless indicated otherwise in a credit line to the material. If material is not included in the article's Creative Commons license and your intended use is not permitted by statutory regulation or exceeds the permitted use, you will need to obtain permission directly from the copyright holder. To view a copy of this license, visit http://creativecommons.org/licenses/by/4.0/. 
therapeutic approach for colon inflammation and tumorigenesis.

Galectin-3-binding protein, LGALS3BP, is a multifunctional secreted glycoprotein involved in immunity, inflammation, and cancer ${ }^{11,12}$. LGALS3BP was shown to downregulate the secretion of IL-4, IL-5, and IL-13 and to activate antiviral responses ${ }^{13,14}$. Previously, we have shown that Lgals3bp inhibits NF-kB inflammatory signaling pathways via TAK1 dephosphorylation and degradation in macrophages and mouse embryonic fibroblasts ${ }^{15}$. Because Lgals3bp expression is increased in cancer patients, its role in cancer has been widely stu$\operatorname{died}^{16-22}$. Although the current evidence regarding Lgals3bp activity in cancer is controversial, Lgals3bp exhibits anti-tumor activity in CRC cells. It suppresses Wnt signals via the ISGylation-dependent ubiquitination of beta-catenin, but is downregulated in advanced CRC tissues $^{19}$. Furthermore, CRC patients exhibiting high Lgals3bp expression in cancer tissues have a lower relapse risk and longer overall survival than those exhibiting low Lgals3bp expression ${ }^{21}$. Therefore, Lgals3bp induction is a therapeutic option for colon cancer. Despite the findings regarding the functions of Lgals3bp in colon cancer, its precise role in colon inflammation and tumorigenesis is unknown. In CRC tumor microenvironments (TMEs), myeloid-derived suppressor cells (MDSCs) promote cancer progression by suppressing $\mathrm{T}$ cells. Various factors are involved in MDSC differentiation and infiltration. Granulocyte-macrophage colony-stimulating factor (GMCSF) and granulocyte colony-stimulating factor (G-CSF) are involved in MDSC accumulation during colon tumorigenesis $^{23,24}$. Galectin-3, the Lgals3bp-binding protein, induces the recruitment of MDSCs into the Lewis lung cancer TME, in response to cisplatin ${ }^{25}$. However, the roles of Lgals3bp in MDSC expansion and TME remain unexplored. Thus, it is important to explore the roles of Lgals3bp in colon inflammation, tumorigenesis, and TME.

In this study, we investigated the roles of Lgals3bp in colonic inflammation and tumorigenesis using mouse models.

\section{Results}

Lgals3bp expression is significantly increased in the colon of mice with DSS-induced colitis

RT-qPCR analysis revealed that Lgals3bp mRNA was ubiquitously expressed in various tissues, including the colon, spleen, liver, and lymph nodes (Fig. S1a). The colon is composed of an epithelial layer and the lamina propria, which is the connective tissue underlying the epithelium. These two fractions were analyzed to further determine the source of Lgals3bp expression in the colon. Western blotting analysis showed that Lgals3bp was expressed on both epithelial cells and the lamina propria (Fig. S1e). Furthermore, Lgals3bp mRNA and protein levels were significantly increased in the colons of DSS-treated mice, as compared to those in the water-fed group (Fig. 1A-E).

\section{Lgals3bp deficiency promotes colitis in mice}

To investigate the potential role of Lgals3bp in colon inflammation, we generated $\mathrm{Lgals}_{3} \mathrm{bp}^{-1-}$ mice. Gene knockout was evaluated by Sanger sequencing (Fig. S1b) and confirmed in the colon and spleen by RT-PCR and western blotting (Fig. S1c, d). First, we challenged wildtype (WT) and Lgals3bp ${ }^{-1-}$ mice with 3\% DSS in drinking water for 7 days, to determine their lethality rate. In comparison with WT mice, Lgals3bp ${ }^{-1-}$ mice exhibited a significantly higher mortality (Fig. 2A). To establish the experimental colitis model, mice were treated with $2.5 \%$ DSS for 7 days and analyzed for colitis severity. Lgals $3 b p^{-1-}$ mice exhibited significantly greater body weight loss, rectal bleeding, and diarrhea than WT mice (Fig. 2B, C) and a significant shortening of the colon and splenomegaly (Fig. 2D, E), which reflects colonic and systemic inflammation, respectively. These results indicated that $L g a l s 3 b p^{-1-}$ mice were more prone to develop severe colitis than WT mice. Because the colon tissue was severely damaged in DSS-treated mice (data not shown), animals were allowed to recover for 1 week after DSS feeding, prior to tissue damage analysis. TUNEL assays showed that colonic apoptosis was increased in Lgals $3 b p^{-1-}$ mice, as compared to that in WT mice (Fig. 2F). Colon tissue alterations were further assessed by histopathological analysis. Histology scores for lymphoid hyperplasia, severity of inflammation, ulceration, and crypt disarray were significantly higher in Lgals $3 b p^{-1-}$ mice than in WT mice (Fig. 2G, H). Overall, Lgals3bp ${ }^{-1-}$ mice were highly susceptible to colitis.

\section{Lgals3bp deficiencies induce inflammatory responses in the colon during colitis}

DSS-induced colitis development is accompanied by the increased production of pro-inflammatory factors ${ }^{26-28}$. To understand the role of Lgals3bp in inflammatory responses during colitis, we measured the expression of pro-inflammatory cytokines, such as IL- 6 , TNF- $\alpha$, and IL$\beta$ using RT-qPCR. The expression of pro-inflammatory cytokines was significantly higher in the colons of Lgals $3 \mathrm{bp}^{-1-}$ mice than in the colons of WT mice, and these results were consistent with those for colitis severity (Fig. 3A); this was confirmed by ELISA (Fig. 3B). The increased production of pro-inflammatory cytokines might exacerbate inflammatory responses and severity of colitis in Lgals3bp ${ }^{-1-}$ mice. As Lgals3bp negatively regulates TAK1-NF- $\mathrm{kB}$ signaling, which is crucial for proinflammatory cytokine expression, we analyzed this pathway in the colons of WT and Lgals3bp ${ }^{-1-}$ mice by western blotting. TAK1 is activated by phosphorylation of Ser and Thr residues ${ }^{29}$; in mice, TAK1 phosphorylation at 
A

B

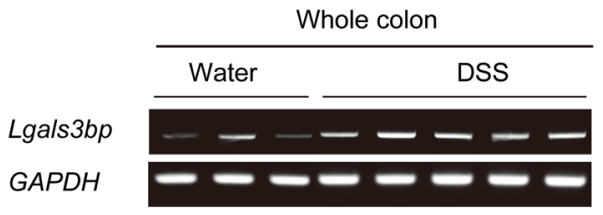

D

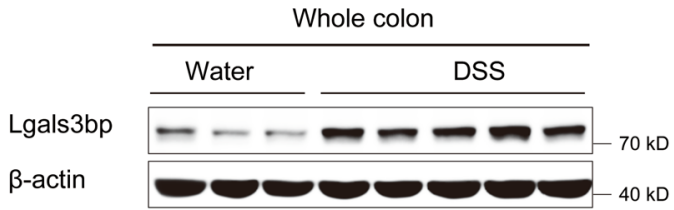

C

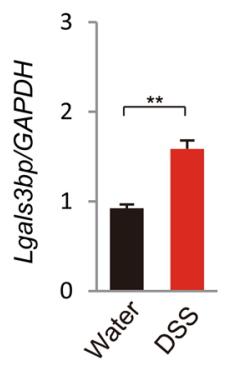

$\mathrm{E}$

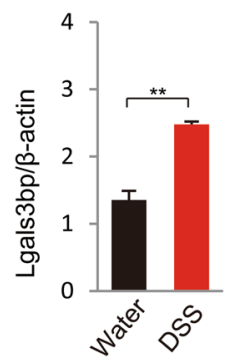

Fig. 1 Elevated Lgals3bp expression in the colon of DSS-induced colitis mice. A Scheme of DSS-induced colitis in WT mice. Mice $(n=5)$ were administrated 2.5\% DSS in drinking water for 7 days and sacrificed on day 7. Waterfed mice $(n=3)$ served as control; colon tissue was collected. B, C RNA was isolated from whole colons, and Lgals3bp and GAPDH expression was analyzed using RT-PCR (B) and RT-qPCR (C). D Protein extracts of the whole colon from WT and Lgals3bp ${ }^{-1-}$ mice were analyzed for the expression of Lgals3bp and $\beta$-actin proteins via western blotting. E Densitometric analysis of band intensities of western blots is shown in (D). Data are presented as means \pm SEM values. ${ }^{* *} p<0.01 ;{ }^{* * *} p<0.001$.

Ser439 ensures complete activation and response to stimuli, such as LPS, TNF- $\alpha$, and IL- $\beta^{30}$. As shown in Fig. 3C, D, TAK1 phosphorylation at Ser439 as well as IкB $\alpha$ and NF-kB p65 phosphorylation were significantly increased in the colons of Lgals3bp ${ }^{-1-}$ mice, as compared to those in WT mice, indicating the hyper activation of TAK1-NF- $\mathrm{B}$ signaling, associated with an enhanced inflammatory response in $L g a l s 3 b p^{-1-}$ mice.

\section{Lgals3bp negatively regulates the TAK1-NF-KB-cytokine axis in epithelial cells}

Next, we assessed the molecular mechanism underlying the effect of Lgals3bp on inflammation in colon epithelial cells. First, we analyzed TLR4-TAK1-NF- $\mathrm{kB}$ signaling in siNC- or siLgals3bp-treated rat intestinal epithelial (RIE) cells by western blotting. As shown in Fig. 4A, LPS stimulation increased TAK1, NF- $k B$, and IkB $\alpha$ phosphorylation in Lgals3bp-knockdown cells, as compared to that in siNC-treated cells. Consistently, the expression of NF$\kappa B$-targets, such as IL- 6 , TNF- $\alpha$, and IL- $\beta$ was significantly higher in Lgals3bp-knockdown cells (Fig. 4B). We investigated whether Lgals3bp overexpression could effectively suppress TLR4-TAK1-NF- $\mathrm{BB}$ pathway activation in RIE cells. As expected, Lgals3bp overexpression inhibited LPS-stimulated TAK1, NF- $\mathrm{kB}$, and IkB $\alpha$ phosphorylation
(Fig. 4C) and IL-6, TNF- $\alpha$, and IL- $\beta$ expression (Fig. 4D). Lgals3bp-mediated NF- $\mathrm{kB}$ suppression was further confirmed by the decreased nuclear translocation of NF- $\mathrm{kB}$ p65 (Fig. 4E). We also analyzed the expression of proinflammatory cytokines in primary colon epithelial cells from WT and Lgals $3 b p^{-1-}$ mice. Upon LPS stimulation, the expression of $I L-6, T N F-\alpha$, and $I L-\beta$ mRNA was significantly higher in $\mathrm{Lgals}_{3} \mathrm{bp}^{-1-}$ cells than that in WT cells (Fig. 4F). These results suggested that Lgals3bp negatively regulated the NF- $\mathrm{kB}$ inflammatory pathway via TAK1 suppression in colon epithelial cells, thus providing protection against colitis.

\section{Lgals $3 b p^{-1-}$ mice are highly susceptible to tumorigenesis in the colon}

Inflammation plays a decisive role in tumorigenesis in the colon. As shown above, Lgals3bp deficiency enhanced colon inflammation. We examined whether it also promoted inflammation-associated tumorigenesis. We induced colon tumorigenesis in WT and Lgals $3 \mathrm{bp}^{-1-}$ mice with AOM/DSS (Fig. 5A). In the AOM/DSS model, mice were subjected to repeated cycles of DSS administration, which resulted in chronic inflammation and promoted AOM-induced neoplastic growth of epithelium $^{26}$. Colitis analysis was performed by monitoring the 
A

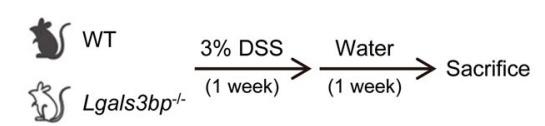

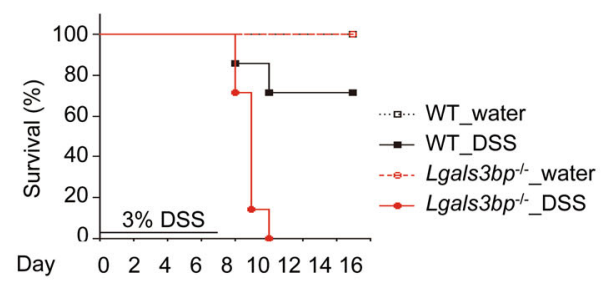

B
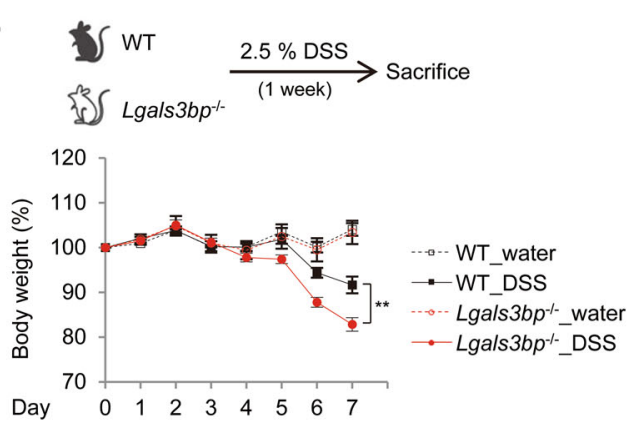

C
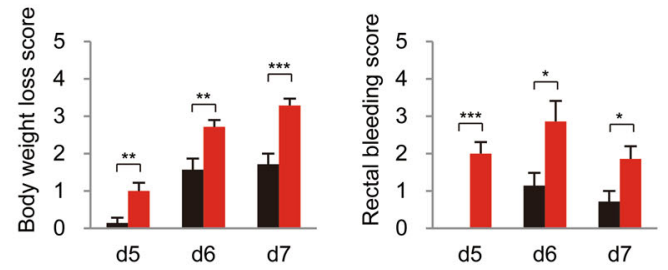

D
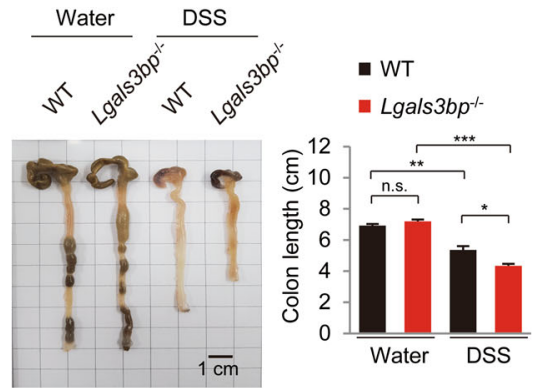

$E$
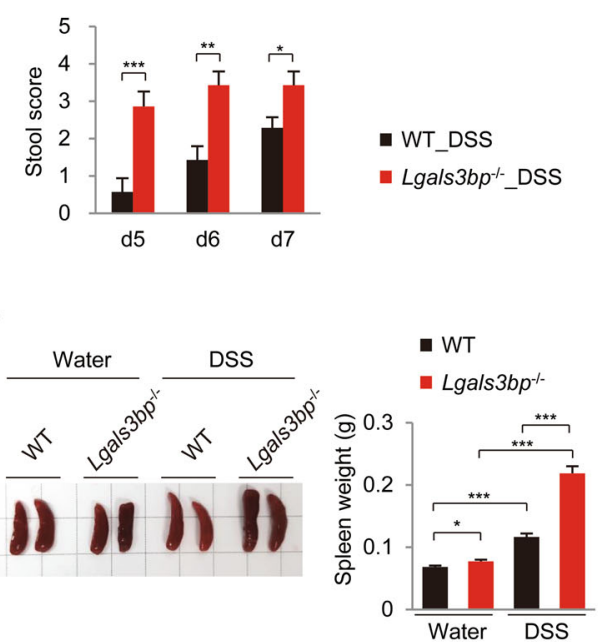

$\mathrm{F}$
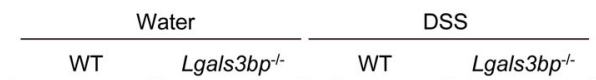

G
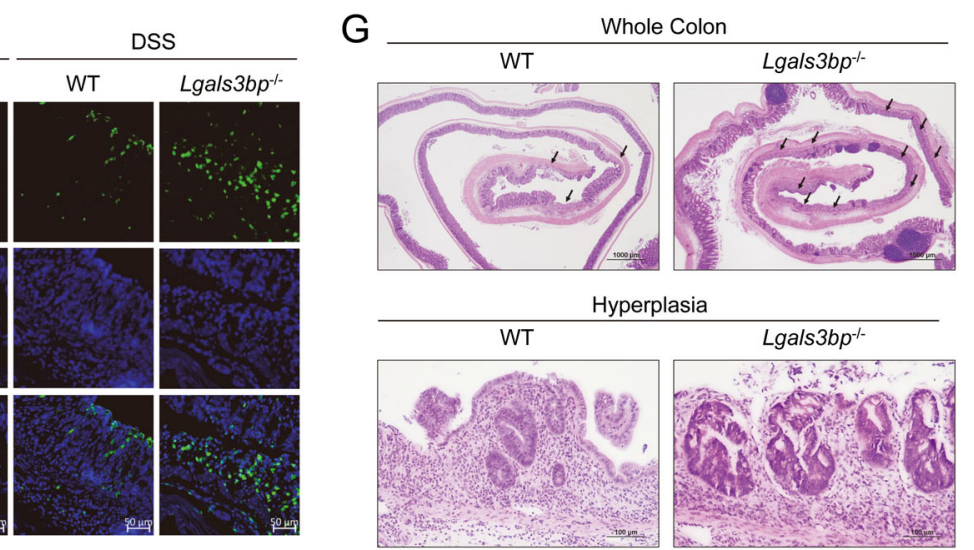

H
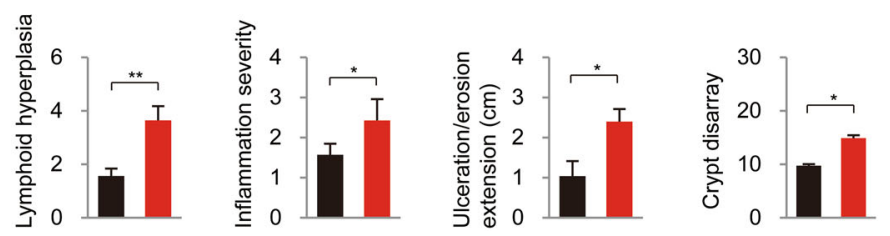

- WT_DSS

- Lgals3bp ${ }^{-1-}$ DSS

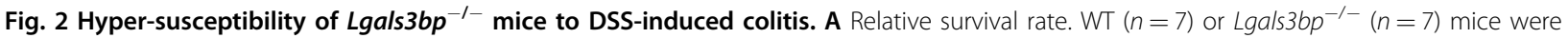
orally administrated 3\% DSS in drinking water for 7 days, followed by regular drinking water. Survival was monitored until day 15 after the start of DSS administration. WT $(n=4)$ or Lgals3bp ${ }^{-1-}(n=4)$ mice provided with water served as controls. B-E WT $(n=7)$ and Lgals3bp ${ }^{-1-}(n=7)$ mice were fed 2.5\% DSS for 7 days. B Body weight change and (C) disease activity index of mice while administering 2.5\% DSS in drinking water. D Colon length and (E) spleen weight measurement at day 7 after 2.5\% DSS administration. (F-H) WT and Lgals3bp ${ }^{-1-}$ mice were fed $2.5 \%$ DSS for 7 days, followed by regular drinking water for 7 days. $\mathbf{F}$ Fluorescent microscopy images of TUNEL stained apoptotic cells in paraffin sections of mouse colons counterstained with DAPI. G Upper: Representative pictures of H\&E-stained colon tissue sections. Arrow indicates histopathological changes. Lower: Representative dysplasia in the colon. $\mathbf{H}$ Histological analysis of colon tissue. H\&E stained sections were scored as described in Materials and Methods. Data are presented as means \pm SEM. ${ }^{*} p<0.05 ;{ }^{* *} p<0.01 ;{ }^{* *} p<0.001 ;{ }^{* * *} p<0.0001$. 
A

$$
\begin{aligned}
& \text {-WT } \\
& \text { - } L \text { gals } 3 b p^{-1-}
\end{aligned}
$$

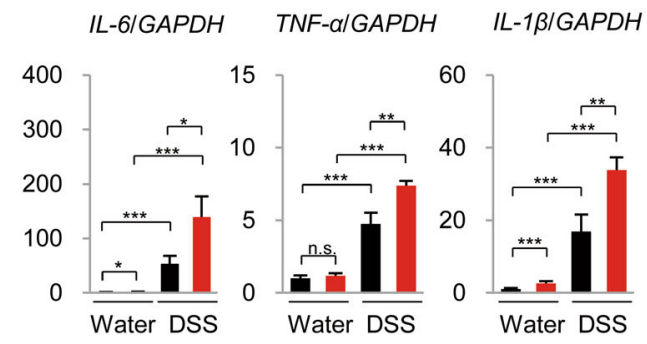

C
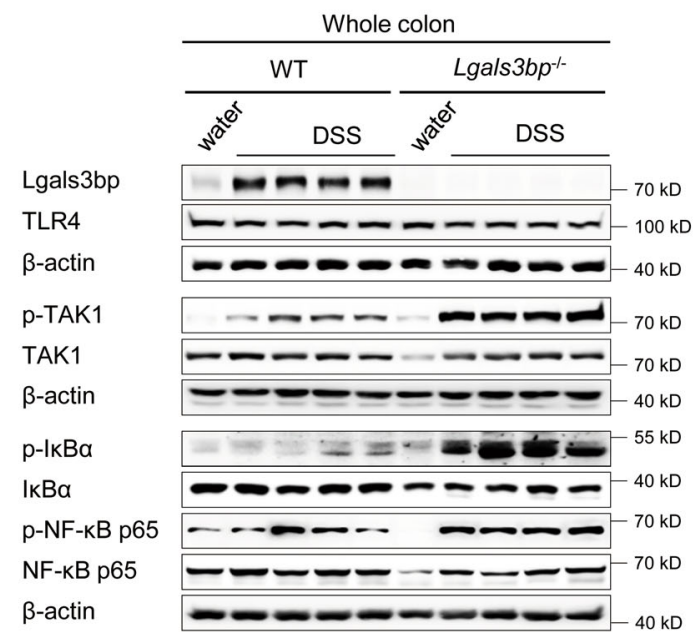

B

- WT

- Lgals $3 b p^{-1-}$

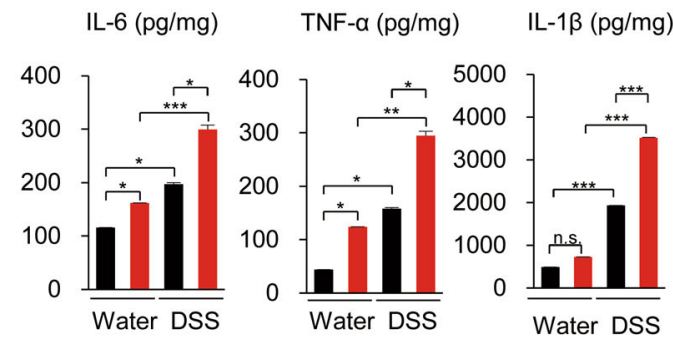

D

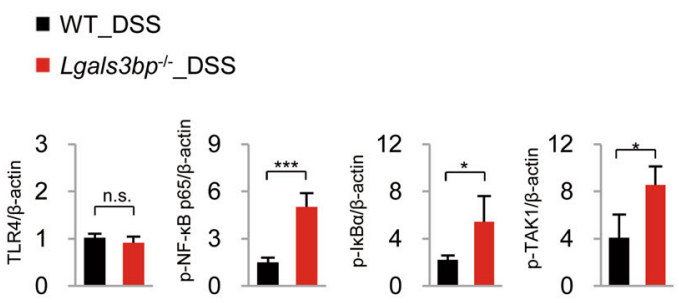

Fig. 3 High inflammatory response in the colon during colitis due to Lgals3bp deficiency. WT $(n=7)$ and Lgals $3 b p^{-/-}(n=7)$ mice were fed with 2.5\% DSS for 7 days and sacrificed on day 7. A RNA was isolated from colons, and the expression of the indicated genes was analyzed by RTqPCR. B The production of IL-6, TNF-a, and IL-1 $\beta$ in the colon homogenate was tested by ELISA. C Protein extracts of the whole colon from WT and Lgals3bp ${ }^{-1-}$ mice were analyzed for the activation of TAK1, NF-KB, and IKB by western blotting. D Densitometric analysis of band intensities of western blots are shown in $(\mathbf{C})$. Data are presented as means \pm SEM values. ${ }^{*} p<0.05 ;{ }^{* *} p<0.01 ;{ }^{* * *} p<0.001 ;{ }^{* * *} p<0.0001$.

body weight; it was significantly reduced in Lgals3bp ${ }^{-1-}$ mice, as compared to WT mice (Fig. 5B). Mice were sacrificed 95 days after AOM administration, to analyze colonic tumor incidence and burden. The number of tumors was significantly greater in $L$ gals $3 b p^{-1-}$ mice than that in WT mice, whereas there was no significant difference in colon length (Fig. 5C, D). Tumor burden was also significantly increased in Lgals3bp ${ }^{-1-}$ mice than that in WT mice (Fig. 5E, F). Together, these results suggested that Lgals3bp deficiency exacerbated tumorigenesis in the colon.

\section{Lgals3bp attenuates TAK1-NF-KB pathway activation in the} colon during tumorigenesis

Next, we investigated whether Lgals3bp inhibited chronic inflammatory responses in the colon during tumorigenesis in a manner similar to that in acute colitis. We analyzed TAK1-NF- $\mathrm{KB}$ activation in colon tissues after the second cycle of DSS administration, which reflects chronic inflammation. In agreement with above-mentioned observations, TAK1, IkB $\alpha$, and NF- $\mathrm{B}$ p 65 phosphorylation was significantly increased in Lgals $3 b p^{-1-}$ mice, as compared to that in WT mice (Fig. 6A, B). Interestingly, total TAK1 levels were also significantly increased in the colon of Lgals $3 \mathrm{bp}^{-1-}$ mice during tumorigenesis. These data supported the observation that Lgals3bp downregulated TAK1-NF- $\mathrm{B}$ B signaling during colonic tumorigenesis.

\section{Increased GM-CSF production in the colons of Lgals $3 b p^{-1-}$ mice promotes tumorigenesis via MDSC expansion and infiltration}

Cytokines and chemokines act as molecular switches for tumorigenesis, by reprogramming the $\mathrm{TME}^{31}$. To investigate critical mediators of colon tumorigenesis in Lgals $3 b p^{-1-}$ mice, we verified cytokine production in mouse colons after the second cycle of DSS 
A

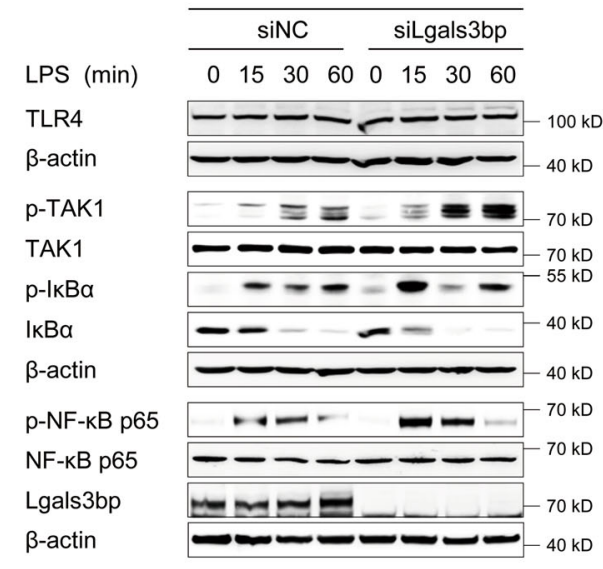

C

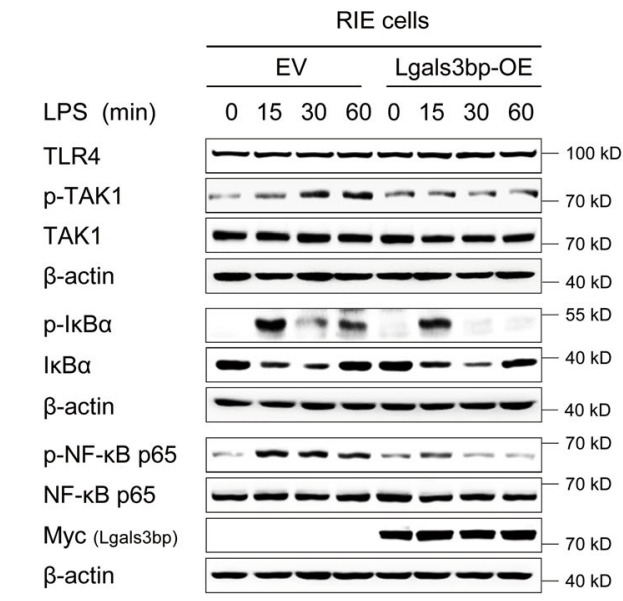

B

- siNC

- siLgals3bp

$$
\text { IL-6/GAPDH TNF- } a / G A P D H \quad I L-1 \beta / G A P D H
$$
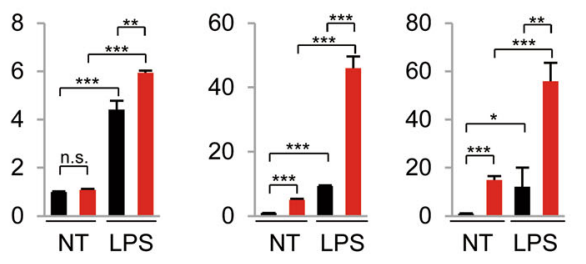

- EV

- Lgals3bp-OE

IL-6/GAPDH TNF- $\alpha / G A P D H \quad I L-1 \beta / G A P D H$
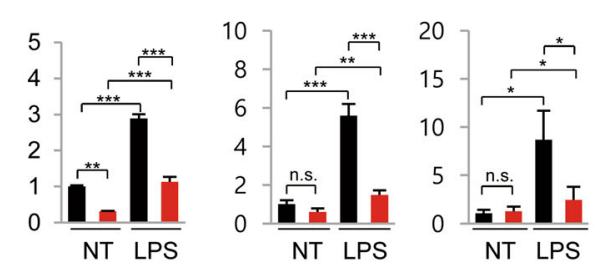

E

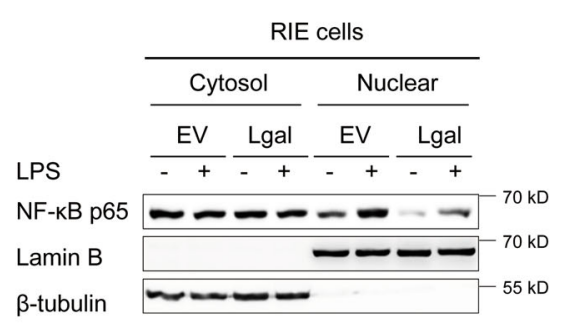

F - wT

- Lgals $3 b p^{-1-}$

$$
\text { IL-6/GAPDH TNF- } \alpha / G A P D H \quad \text { IL-1ß/GAPDH }
$$

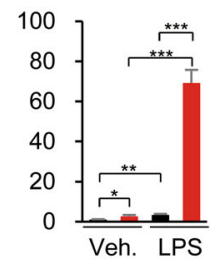

Fig. 4 Lgals3bp-induced downregulation of NF-KB-inflammatory cytokine axis via TAK1 suppression in epithelial cells. A-B Lgals3bpknockdown RIE cells were treated with LPS $(100 \mathrm{ng} / \mathrm{ml})$ for indicated periods. A Whole cell lysates were analyzed for the activation of TLR4, TAK1, IKB, and NF-KB via western blotting. B RNA was isolated from LPS-treated RIE cells, and the expression of the indicated genes was analyzed by RT-gPCR. C, D Lgals3bp-overexpressed RIE cells were treated with LPS (100 ng/ml) for indicated periods. C Whole cell lysates were analyzed for TLR4, TAK1, IKB, and NF-KB activation by western blotting. D RNA was isolated from LPS-treated RIE cells, and IL-6, TNF- $a$, and IL-1 $\beta$ gene expression levels were analyzed by RT-qPCR. E Lgals3bp-overexpressed RIE cells were treated with LPS (100 ng/ml) for 15 min. The expression level of NF-kB p65 was analyzed in the cytoplasmic and nuclear fractions. $\beta$-tubulin and lamin B were used as the loading controls for the cytosol and nucleus, respectively. F Primary colon epithelial cells were isolated from WT and $\mathrm{Lgals}_{3} \mathrm{bp}^{-/-}$mice, and treated with LPS $(100 \mathrm{ng} / \mathrm{ml})$ for $3 \mathrm{~h}$. The expression of indicated genes was analyzed by RT-qPCR. Data are presented as means \pm SEM values. ${ }^{*} p<0.05 ;{ }^{* *} p<0.01 ;{ }^{* * *} p<0.001 ;{ }^{* * *} p<0.0001$.

administration using the Luminex cytokine quantification assay. Surprisingly, even though IL- 6 , TNF- $\alpha$, and IL- $\beta$ production tended to increase in Lgals $3 b p^{-1-}$ mice, the difference observed with WT mice was not statistically significant. Simultaneously, the production of GM-CSF, which is normally low and tightly regulated, was 


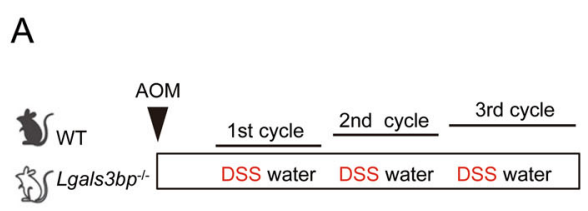

C

B
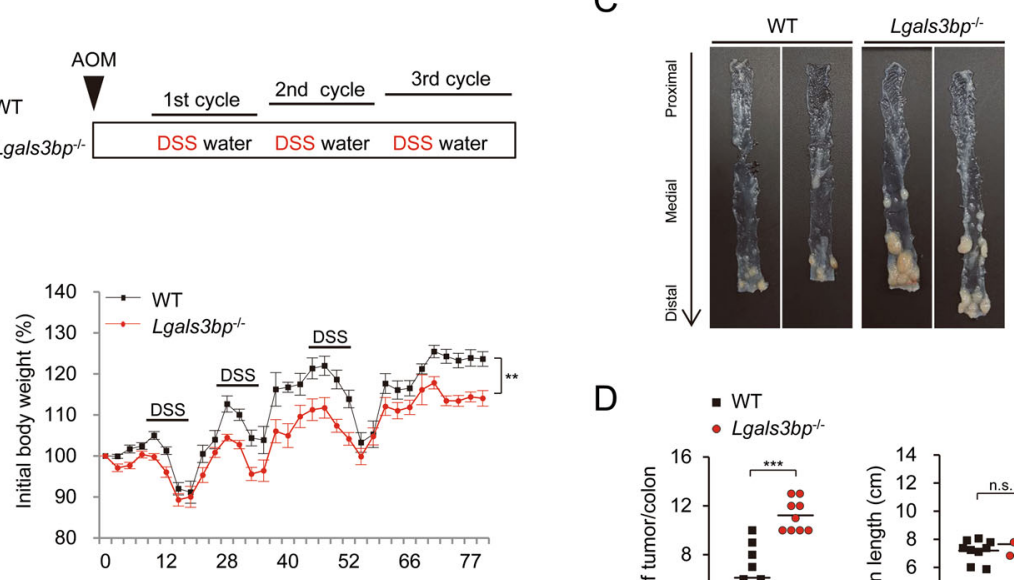

D

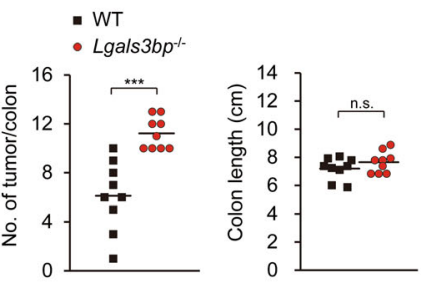

E

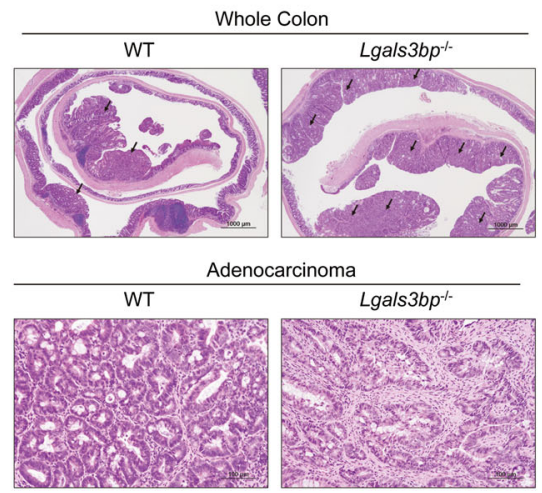

$\mathrm{F}$

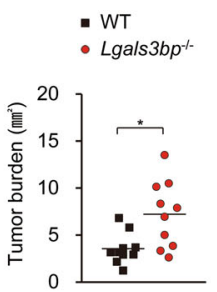

Fig. 5 Hyper-susceptibility of $\mathbf{L g a l s} 3 b p^{-1-}$ mice to colon tumorigenesis. A Schematic overview of the AOM/DSS protocol of colon tumorigenesis model. In brief, WT $(n=9)$ and Lgals3bp ${ }^{-1-}(n=9)$ mice were treated intraperitoneally with AOM $(10 \mathrm{mg} / \mathrm{kg})$. After 5 days, mice were treated with 2.5\% DSS in drinking water for 1 week. Thereafter, mice were fed with regular water for 2 weeks and subjected to two more DSS administration cycles. Mice were sacrificed at day 95 following AOM injection. B Body weight was monitored during AOM/DSS-induced tumorigenesis. C Representative image of tumor-bearing colons from WT and $\mathrm{Lgals} 3 \mathrm{bp} \mathrm{p}^{-1-}$ mice. D The tumor number per colon was counted and colon length was measured. E Upper: Representative picture of H\&E stained tumor-bearing colons. Arrows indicate tumor lesions. Lower: Representative adenocarcinoma in the colon $\mathbf{F}$ Tumor burden was measured based on histological analysis. The solid line within the graph represents the mean value. ${ }^{* *} p<0.01$.

significantly increased in the colons of $\mathrm{Lgals}_{3} \mathrm{bp}^{-1-}$ mice, whereas that of the related macrophage CSF and granulocyte CSF was not increased (Fig. 6C). We further confirmed the negative regulation of GM-CSF by Lgals3bp in chronically inflamed colons and primary colon epithelial cells by RT-qPCR (Fig. S2a, b). These data supported the idea that Lgals3bp downregulated GM-CSF production in chronically inflamed colons during tumorigenesis.

As GM-CSF is involved in MDSC accumulation during tumorigenesis in the colon, we analyzed the MDSC population in the mouse spleens after the second cycle of DSS administration. Consistent with the high level of GM-CSF, the MDSC population was significantly increased in $\mathrm{Lgals}_{3} \mathrm{bp}^{-1-}$ mice, as compared to that in
WT mice (Fig. 6D). To verify tumor infiltration of MDSCs, we analyzed the MDSC population in colon cancer tissues and spleens at the endpoint of AOM/DSS model. As shown in Fig. 6E, the ratios of tumor-infiltrated and splenic MDSCs were significantly increased in Lgals $3 b p^{-1-}$ mice. As expected, the MDSC population was positively correlated with GM-CSF production (Fig. $6 \mathrm{~F}$ ). Overall, these findings suggested that increased GMCSF production in the colon of Lgals $3 b p^{-1-}$ mouse promoted tumorigenesis via MDSC accumulation. We, therefore, propose that the elevated production of GMCSF in Lgals $3 b p^{-1-}$ mice due to the increased activation of TAK1-NF- $\mathrm{KB}$ signaling leads to MDSC accumulation and stimulation of tumorigenesis in the colon (Fig. 7). 
A

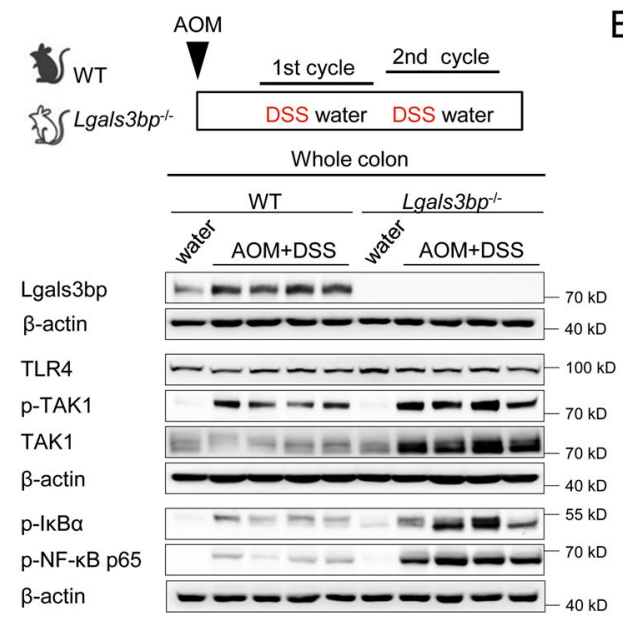

B

- WT_AOM+DSS

- Lgals $3 b p^{-1}{ }_{-}$AOM+DSS
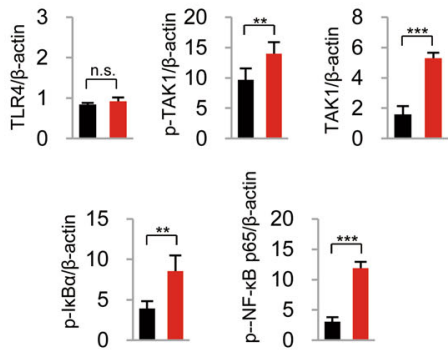

C
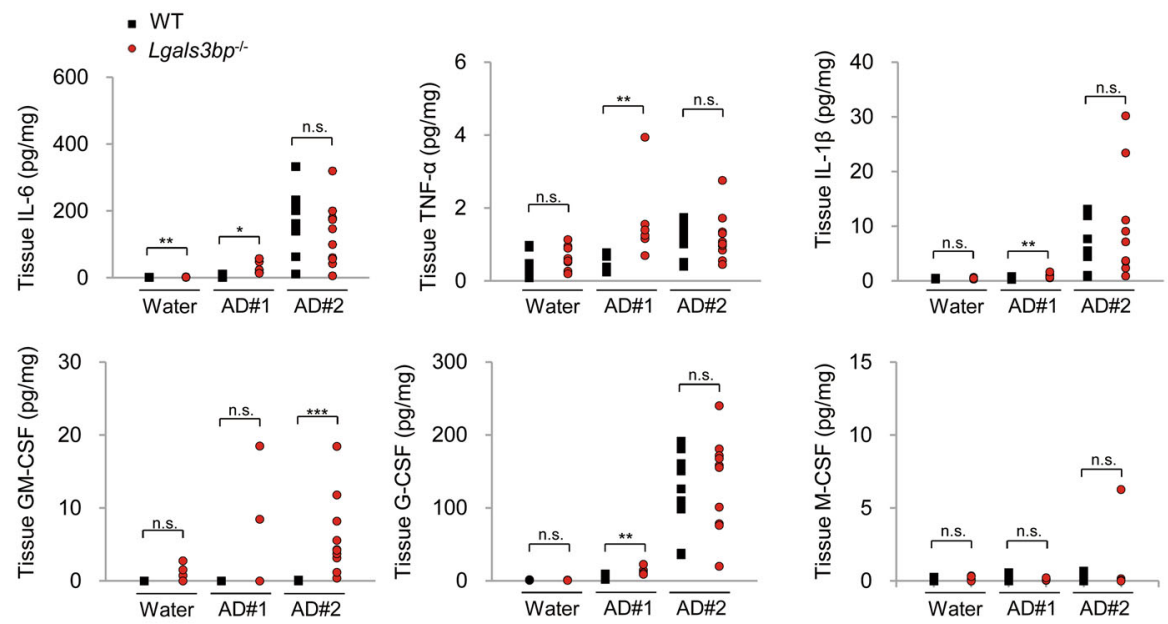

D

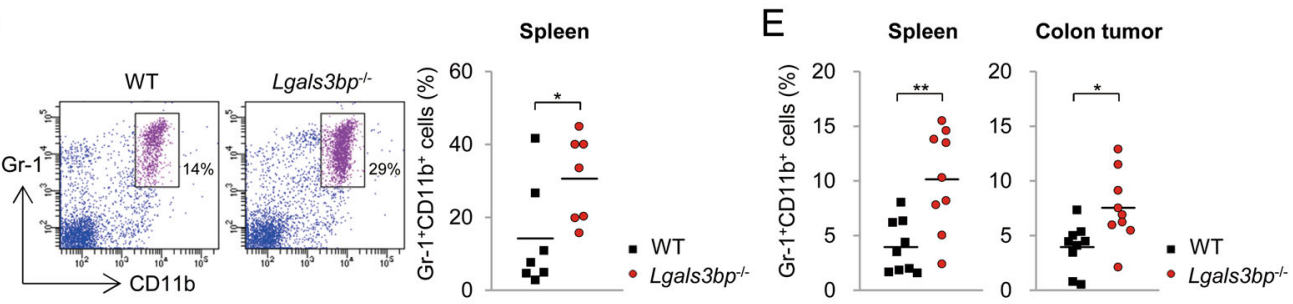

$\mathrm{F}$

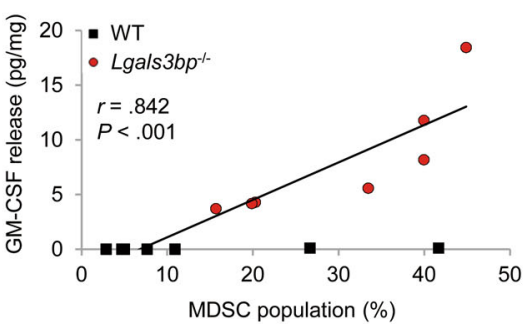

Fig. 6 (See legend on next page.)

\section{Discussion}

We have shown that Lgals3bp negatively regulates colonic inflammation and tumorigenesis by suppressing
TAK1-NF- $\mathrm{B}$ signaling in the colon epithelium. This suggests that Lgals3bp exhibits protective activities against inflammation and tumorigenesis in the colon. 
(see figure on previous page)

Fig. 6 Lgals3bp deficiency-induced exacerbation of colon tumorigenesis via GM-CSF-mediated MDSC accumulation. A WT and $L g a / s 3 b p^{-1-}$ mice were treated with AOM $(10 \mathrm{mg} / \mathrm{kg})$. After 5 days of AOM administration, WT $(n=9)$ and $\mathrm{Lgals}_{3} \mathrm{bp} \mathrm{p}^{-1-}(n=9)$ mice were subjected to two DSS cycles. Mice were sacrificed at day 50 following AOM injection. WT $(n=6)$ and Lgals $3 b p^{-1-}(n=6)$ mice were subjected to one DSS cycle. Mice were sacrificed at day 12 following AOM injection. WT $(n=9)$ or Lgals3bp $p^{-1-}(n=9)$ mice provided with water served as controls. Protein extracts of colons from WT and Lgals3bp ${ }^{-1-}$ mice were analyzed for TAK1, NF-KB, and IKB activation via western blotting. B Densitometric analysis of band intensity of western blots is shown in (A). C Production levels of cytokines and chemokines in colons were determined by the Luminex assay. Water AD\#1, and AD\#2 indicate water feeding group, AOM with one DSS cycle group, and AOM with two DSS cycles group. D The total spleen cells were isolated and the MDSC population $\left(\mathrm{Gr}-1^{+} \mathrm{CD} 11 \mathrm{~b}^{+}\right)$was examined by flow cytometry. E AOM/DSS model mice were sacrificed at day 95 following $\mathrm{AOM}$ injection. The total cells of the spleen and colon tumor were isolated, and the population of MDSCs $\left(\mathrm{Gr}-1^{+} \mathrm{CD} 11 \mathrm{~b}^{+}\right.$) was examined by flow cytometry. $\mathbf{F}$ The correlation between MDSC and GM-CSF abundance in the colon from tumor-bearing mice. The solid line within the graph represents the mean value. Data are presented as means \pm SEM values. ${ }^{*} p<0.05 ;{ }^{*} p<0.01 ;{ }^{* * *} p<0.001$.

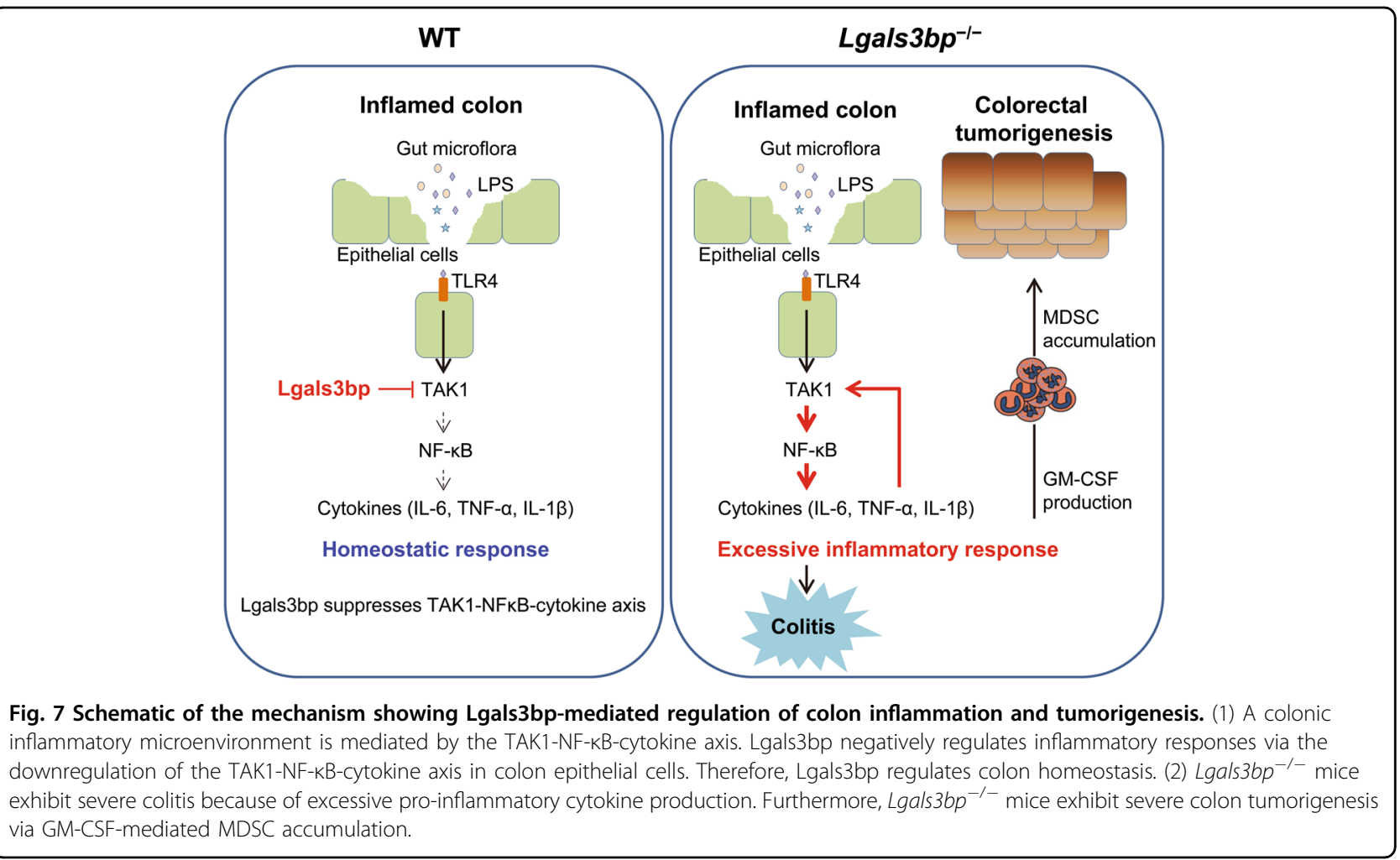

The involvement of luminal flora and TLR signaling is a unique aspect of colonic inflammation and tumorigenesis $^{1,32}$. LPS is abundantly released by the colonic bacteria and interacts with TLR4, which activates the TAK1-NF$\kappa \mathrm{B}$-cytokine axis, and ultimately promotes inflammation and tumorigenesis in the colon. Lgals3bp negatively regulated these signals through TAK1 suppression. NF- $\mathrm{KB}$ reportedly mediated TNF- $\alpha$-induced Lgals $3 b p$ mRNA expression in breast cancer cells ${ }^{33}$. In agreement with this result, Lgals3bp was upregulated in the NF- $\mathrm{kB}$-activated inflamed colon. Investigating the phenotypes of Lgals3bp knockout is crucial for understanding its function in the colon. Lgals $3 b p^{-1-}$ mice were highly susceptible to inflammation and tumorigenesis in the colon. This study furthers our understanding of the negative regulation of inflammation in the colon, where Lgals3bp inhibits TAK1-NF- $k B$-cytokine signaling to prevent excessive inflammation and tumorigenesis.

Epithelial cells, the major cell type in the colon, act as gatekeepers of gut immune homeostasis and provide a barrier against luminal bacterial flora. Our results show that levels of Lgals3bp, expressed in colon epithelial cells, are further increased by inflammation. Macrophages provide robust inflammatory responses via TLR activation in the lamina propria of the gut ${ }^{26,34}$. Given that Lgals3bp is also expressed in macrophages ${ }^{15}$, we conclude that Lgals3bp suppresses inflammatory responses in both epithelial cells and gut macrophages. 
TAK1 has emerged as a therapeutic target for inflammatory disorders, fibrosis, and cancer ${ }^{9}$. TAK1 inhibition suppresses inflammation and fibrosis in the colon, kidney, and lung ${ }^{30,35,36}$ and inhibits the in vivo growth of KRASmutated colon cancer cells and pro-survival signaling in breast cancer cells ${ }^{37,38}$. Furthermore, TAK1 inhibitors reduce chemoresistance in pancreatic, ovarian, and esophageal cancer cells ${ }^{39-41}$. As TAK1 is a key kinase linking exogenous stimuli and cellular responses, cancer cells utilize it to adapt to the TME. TAK1 targeting is effective in inhibiting breast cancer lung metastasis, via the suppression of IL-1 secretion ${ }^{42}$. With the recent interest in TAK1 as a target for inflammatory diseases and cancer, selective and orally active inhibitors, including LYTAK1 and oxindole derivatives, are currently under development ${ }^{9}$. Cellular Lgals3bp directly interacts with TAK1, and reduces protein stability and the binding affinity between TAK1 and its adaptor proteins ${ }^{15}$. Other studies suggested that Lgals3bp exhibited antiviral activity, and reported about the interaction between Lgals3bp and TAK $1^{14}$. Here, we revealed that Lgals3bp is an endogenous negative regulator of TAK1 in the colon and suggest that Lgals3bp induction or use of the Lgals3bp-based peptide might be a potential therapeutic approach for IBD and colon cancer. Further studies are necessary to map the region of Lgals3bp responsible for TAK1 interaction and suppression.

In the TME, MDSCs contribute to tumorigenesis and cancer immune evasion by suppressing the anti-tumor functions of $\mathrm{T}$ cells ${ }^{43}$. Therefore, it is important to identify factors influencing MDSC differentiation, recruitment, and activation. GM-CSF has been linked to MDSC generation in tumorigenesis, and elevated GM-CSF production has been observed in the mucosa of IBD patients and in a mouse model of colitis ${ }^{23,44}$. Colon epithelial cells are a major cellular source of GM-CSF production in the AOM/DSS model. GM-CSF is significantly increased in the chronic inflamed colon and its blockade suppresses MDSC activation and cancer development ${ }^{23}$. Here, we found that Lgals3bp deficiency promoted colonic tumorigenesis through an increase in GM-CSF and accumulation of MDSCs. Because GM-CSF also promoted tumorassociated macrophage (TAM) activation ${ }^{45}$, it should be investigated whether LGALS3BP suppresses pro-tumor cells, including TAMs, through the downregulation of GM-CSF and other cytokines in the TME.

Although LGALS3BP was originally identified in cancer cells, its role in tumorigenesis remains unclear. LGALS3BP reportedly has both negative and positive influences on various cancer prognoses. For example, though high serum LGALS3BP levels are associated with poor clinical outcomes in patients with breast, hepatocellular, and non-small cell lung carcinoma ${ }^{16,46,47}$, high LGALS3BP expression in tumor tissues is associated with favorable outcomes in patients with Ewing's sarcoma and $\mathrm{CRC}^{21,22}$. Thus, our results suggest that Lgals3bp suppresses colonic tumorigenesis via TAK1 downregulation. Based on the various functions of TAK1, LGALS3BP might inhibit tumorigenesis and cancer progression by suppressing tumor cell proliferation, metastasis, and anticancer drug resistance. Therefore, further studies are required to investigate the role of LGALS3BP in various aspects of cancer.

In conclusion, this study demonstrates that Lgals3bp is a physiological regulator of inflammation and tumorigenesis in the colon. We provided molecular evidence that Lgals3bp-mediated protection against colonic inflammation and tumorigenesis reflects its unique function of inhibiting TAK1-NF-kB signaling and that Lgals3bp deficiency advances colon tumorigenesis by increasing GMCSF production and MDSC accumulation. Therefore, the Lgals3bp-mediated negative regulation of TAK1 might be a key target for colon cancer immunotherapy.

\section{Material and methods \\ Reagents and antibodies}

Lipopolysaccharide (LPS) from Escherichia coli 055:B5 (L2880) and azoxymethane (AOM; A5486) were purchased from Sigma-Aldrich (St. Louis, MO, USA). Dextran sodium sulfate (DSS; 160110) was purchased from MP Biomedicals (Solon, OH, USA). Other chemical reagents were obtained from Sigma-Aldrich.

\section{Cell culture and transfection}

Rat intestinal epithelial (RIE) cells (generously gifted by Prof. Young Eun Joo) were cultured in Dulbecco's modified Eagle's medium (Invitrogen, Carlsbad, CA, USA) supplemented with $10 \%$ fetal bovine serum (FBS) and 1\% penicillin-streptomycin (Invitrogen). Lgals3bp and negative control siRNAs were purchased from Bioneer Corp. (Daejeon, Republic of Korea). siRNA and DNA were transfected using Lipofectamine RNAiMAX (Invitrogen; 13778150) and Lipofectamine 3000 (Invitrogen, L3000015), respectively according to the manufacturer's protocol. For transient transfection, RIE cells were grown to $70 \%$ confluence in a 6-well plate. Lipofectamine 3000 $(3.75 \mu \mathrm{L})$ was diluted and mixed with $150 \mu \mathrm{L}$ of OptiMEM (Invitrogen; 11058021). Plasmid DNA $(2.5 \mu \mathrm{L})$ was diluted in $150 \mu \mathrm{L}$ of Opti-MEM and then $5 \mu \mathrm{L}$ of P3000 was added to it. The two mixtures were then combined and incubated for $5 \mathrm{~min}$. The transfection mixture was added to RIE cells. For siRNA-mediated knockdown of Lgals3bp, RIE cells were transfected with $30 \mathrm{nM}$ of either the control or Lgals3bp siRNA using RNAiMAX for $48 \mathrm{~h}$. Briefly, siRNA and RNAiMAX were diluted in OptiMEM. The two mixtures were then combined and incubated for $5 \mathrm{~min}$. The RIE cells were treated with the transfection mixture. The sequences of siRNAs targeting 
Lgals3bp were as follows: sense: $5^{\prime}$-GAGACUUCCUCA GGUACUUtt-3'; antisense: 5'-AAGUACCUGAGGAAG UCUCtt- $3^{\prime}$.

\section{Mice and colitis and tumorigenesis models \\ Lgals3bp $^{-1-}$ C57BL/6N mice were generated using the CRISPR-Cas9 genome editing system (Macrogen, Inc., Seoul, Korea) as described previously ${ }^{15}$. All experimental mice were housed in a specific, pathogen-free facility. All animal protocols were approved by The Chonnam National University Medical School Research Institutional Animal Care and Use Committee (IACUC; CNU-IACUC- $\mathrm{H}$-2017-50). Experiments were conducted in accordance with the IACUC guidelines. The colitis model was established by applying 2.5\% DSS to the drinking water for 7 days. Colon tumorigenesis was induced using the AOM/ DSS model, as described previously ${ }^{26}$. C57BL/6N mice (6-8 weeks old, male) were used in all experiment.}

\section{Colitis index scoring}

The criteria for assessing colitis severity included body weight loss, stool consistency, and rectal bleeding. Body weight loss scores (0-4) were as follows: 0 , none; $1,1-5 \%$ loss; 2, 6-10\% loss; 3, 11-20\% loss; 4, >20\% loss. Stool consistency was scored as follows: 0 , normal; 2 , soft (loose); 3 , diarrhea. Rectal bleeding was scored as follows: 0 , none; 1 , occult bleeding; 3 , red; 4 , dark red; 5 , gross bleeding.

\section{TUNEL and histopathological analysis}

Colons were fixed in $4 \%$ paraformaldehyde and embedded in paraffin. TUNEL assays were performed using the Dead-End fluorometric TUNEL system (Promega, Madison, WC, USA; G3250), according to the manufacturer's protocol. Colon sections were stained with hematoxylin and eosin (H\&E). Histopathological scoring was performed in a blinded fashion by an experienced pathologist [Lee KH]. Histological scoring was based on four parameters. The severity of lymphoid follicles and hyperplasia was scored as follows: 0 , normal; 1 , mild; 2 , moderate; 3 , severe. Inflammation severity was scored as follows: 0 , rare inflammatory cells in lamina propria; 1 , increased numbers of leukocytes in lamina propria; 2, confluence of inflammatory cells extending into the submucosa; 3, transmural extension of the inflammatory infiltrate. Areas of epithelial loss, indicative of ulcers/ erosion and changes in the crypt architecture, including flattening atypia and glandular complexity, were measured within colon sections.

\section{Isolation of colonic epithelial cells and lamina propria}

To isolate colonic epithelial cells, colons were sectioned into small pieces and digested in Digestion Buffer 1 (RPMI 1640 containing 5\% FBS, $5 \mathrm{mM}$ EDTA, and $1 \mathrm{mM}$ dithiothreitol) at $37^{\circ} \mathrm{C}$. The remaining tissues were further digested in Digestion Buffer 2 (RPMI 1640 containing $5 \%$ FBS, collagenase, and DNase 1 ) at $37^{\circ} \mathrm{C}$ for $50 \mathrm{~min}$. The homogenate was filtered with a $70 \mu \mathrm{m}$ Nylon cell strainer (BD Biosciences, Bedford, MA, USA), washed with Washing Buffer 1 (ice-cold PBS containing 0.2\% EDTA), and re-suspended in Washing Buffer 2 (ice-cold PBS containing 3\% FBS and 0.2\% EDTA).

\section{Flow cytometry}

Cells isolated from tumors, spleens, and red blood cells were removed with lysis buffer (Sigma; R7757). Cell suspensions in FACS buffer (1\% BSA/PBS) were blocked for 15 min with CD16/32 antibodies (eBioscience, INC. San Diego, CA). Cells were stained for $30 \mathrm{~min}$ at $4{ }^{\circ} \mathrm{C}$ with fluorescently-conjugated $\mathrm{CD} 11 \mathrm{~b}$ and $\mathrm{Gr}-1$ antibodies. Samples were analyzed using FACScanto (BD PharMingene) and FlowJo Software (Treestar, Ashland, OR).

\section{Nuclear and cytoplasmic fractionation}

Nuclear and cytoplasmic fractions were prepared using the NE-PER Nuclear and Cytoplasmic Extraction Reagent (Thermo Fisher Scientific, Waltham, MA, USA; 78835), according to the manufacturer's instructions.

\section{Western blot analysis}

Whole cell lysates were prepared with a protein extraction reagent (Thermo Fisher Scientific; 78501). Equal protein amounts were boiled, separated by SDSPAGE, and transferred onto a PVDF membrane. Membranes were blocked using SuperBlock ${ }^{\mathrm{TM}}$ T20 blocking buffer (Thermo Fisher Scientific; 37515) and incubated overnight at $4{ }^{\circ} \mathrm{C}$ with primary antibodies (Supplementary Table 1). Horseradish peroxidase-conjugated secondary antibodies were used to probe the membranes for $1 \mathrm{~h}$ at $25^{\circ} \mathrm{C}$, and the membranes were visualized using a lowlight imaging system (LAS-4000 mini; FUJIFILM Medical Systems). Band intensities were quantified using the Multi-Gauge 3.0 software.

\section{PCR and real-time quantitative (RT-q) PCR}

Total RNA was isolated using the Hybrid-R reagent (305-101; GeneAll Biotechnology, Seoul, Korea). Equal amounts of cDNA were synthesized with $500 \mathrm{ng}$ of sample RNA, using the GoScript Reverse Transcription Mix (Promega; A2791). PCR was performed using GoTaq DNA polymerase (Promega; M300F) and gene-specific primer pairs. RT-qPCR was performed using Quantifast SYBR Green PCR Master Mix (Qiagen, Hilden, Germany; 204054) on a RotorGene 3000 (Qiagen) machine. Primers are listed in Supplementary Table 2.

\section{Cytokine analysis}

Cytokine levels in tissue homogenates were measured using commercial enzyme-linked immunosorbent assay 
(ELISA) kits for mouse IL-6 (51-25632E), IL-1 $\beta$ (51-26662E), and TNF- $\alpha$ (51-26732E) (BD Biosciences, San Jose, CA, USA). Alternatively, cytokines and chemokines were quantified with the multiplex assay (EMD Millipore, MCYTOMAG-70K), according to the manufacturer's instructions.

\section{Statistical analysis}

Statistical analysis was performed using SPSS 12.0 (SPSS, Inc., Chicago, IL, USA). Statistical significance was assessed by Mann-Whitney $U$ test. Survival analysis was performed using the Kaplan-Meier method. Statistically significant were considered when $P<0.05$.

\section{Acknowledgements}

This work was supported by the Basic Science Research Program (NRF2018R1D1A1B07049785) through the National Research Foundation of Korea (NRF), funded by the Ministry of Education. This work was supported by NRF grants (NRF-2020R1A2C2009439, NRF-2018R1A5A2024181) and the Bio \& Medical Technology Development Program (NRF-2019M3E5D1A02068082, NRF-2020M3A9G3080281) through the NRF grant funded by the Korean government (MSIT). We wish to thank Editage (www.editage.co.kr) for English language editing.

\section{Author details \\ 'Department of Hematology and Oncology, Chonnam National University Medical School and Hwasun Hospital, Hwasun, Republic of Korea. ${ }^{2}$ Immunotherapy Innovation Center, Chonnam National University Medical School and Hwasun Hospital, Hwasun, Republic of Korea. ${ }^{3}$ Combinatorial Tumor Immunotherapy MRC Center, Chonnam National University Medical School, Hwasun, Republic of Korea. ${ }^{4}$ Department of Pathology, Chonnam National University Medical School and Hwasun Hospital, Hwasun, Republic of Korea. ${ }^{5}$ Department of Pharmacology and Toxicology, Sylhet Agricultural University, Sylhet, Bangladesh}

\section{Author contributions}

S.H.C., H.J.S., E.G.S., and I.J.C. conceived and designed the study. S.H.C., H.J.S., E. G.S., M.R.P., and J.N.C. performed the experiments. S.H.C., H.J.S., E.G.S., W.K.B., J.E. H., K.H.L., and I.J.C. analyzed and interpreted the data. E.G.S. and M.R.A. wrote the draft paper. E.G.S. and I.J.C. obtained the research funding. All authors have read and approved the final paper. S.H.C. and H.J.S. contributed equally to this work.

\section{Data availability}

The datasets used and analyzed during the current study are available from the corresponding author on reasonable request.

\section{Conflict of interest}

The authors declare no competing interests.

\section{Publisher's note}

Springer Nature remains neutral with regard to jurisdictional claims in published maps and institutional affiliations.

Supplementary information The online version contains supplementary material available at https://doi.org/10.1038/s41420-021-00447-7.

Received: 2 November 2020 Revised: 18 February 2021 Accepted: 8 March 2021

Published online: 06 April 2021

\footnotetext{
References

1. Ullman, T. A. \& Itzkowitz, S. H. Intestinal inflammation and cancer. Gastroenterology 140, 1807-1816 (2011).
}

2. Lutgens, M. W. et al. Declining risk of colorectal cancer in inflammatory bowel disease: an updated meta-analysis of population-based cohort studies. Inflamm. Bowel Dis. 19, 789-799 (2013).

3. Brody, H. Colorectal cancer. Nature 521, S1 (2015).

4. Miller, K. D. et al. Cancer treatment and survivorship statistics, 2016. CA Cancer J. Clin. 66, 271-289 (2016).

5. Ben-Neriah, Y. \& Karin, M. Inflammation meets cancer, with NF-kappaB as the matchmaker. Nat. Immunol. 12, 715-723 (2011).

6. Liu, T., Zhang, L., Joo, D. \& Sun, S. C. NF-kappaB signaling in inflammation. Signal Transduct. Target Ther. 2, e17023 (2017).

7. Sato, S. et al. Essential function for the kinase TAK1 in innate and adaptive immune responses. Nat. Immunol. 6, 1087-1095 (2005).

8. Yamaguchi, $K$. et al. Identification of a member of the MAPKKK family as a potential mediator of TGF-beta signal transduction. Science 270, 2008-2011 (1995).

9. Sakurai, H. Targeting of TAK1 in inflammatory disorders and cancer. Trends Pharm. Sci. 33, 522-530 (2012).

10. Pekalski, J. et al. Spontaneous NF-kappaB activation by autocrine TNFalpha signaling: a computational analysis. PLOS ONE 8, e78887 (2013).

11. Koths, K., Taylor, E., Halenbeck, R., Casipit, C. \& Wang, A. Cloning and characterization of a human Mac-2-binding protein, a new member of the superfamily defined by the macrophage scavenger receptor cysteine-rich domain. J. Biol. Chem. 268, 14245-14249 (1993).

12. Silvestri, B. et al. Differential effect on TCR:CD3 stimulation of a 90-kD glycoprotein (gp90/Mac-2BP), a member of the scavenger receptor cysteine-rich domain protein family. Clin. Exp. Immunol. 113, 394-400 (1998).

13. Kalayci, $\mathrm{O}$. et al. Role of $90 \mathrm{~K}$ protein in asthma and TH2-type cytokine expression. Ann. Allergy Asthma Immunol. 93, 485-492 (2004).

14. $\mathrm{Xu}, \mathrm{G}$. et al. Inducible LGALS3BP/90K activates antiviral innate immune responses by targeting TRAF6 and TRAF3 complex. PLoS Pathog. 15, e1008002 (2019).

15. Hong, C. S. et al. Gal-3BP Negatively Regulates NF-kappaB Signaling by Inhibiting the Activation of TAK1. Front. Immunol. 10, 1760 (2019).

16. lacobelli, S. et al. Prognostic value of a novel circulating serum $90 \mathrm{~K}$ antigen in breast cancer. Br. J. Cancer 69, 172-176 (1994)

17. Rea, A. et al. 90k is a serum marker of poor-prognosis in non-hodgkinslymphoma patients. Oncol. Rep. 1, 723-725 (1994).

18. Kunzli, B. M. et al. Influences of the lysosomal associated membrane proteins (Lamp-1, Lamp-2) and Mac-2 binding protein (Mac-2-BP) on the prognosis of pancreatic carcinoma. Cancer 94, 228-239 (2002).

19. Lee, J. H. et al. Glycoprotein $90 \mathrm{~K}$, downregulated in advanced colorectal cancer tissues, interacts with CD9/CD82 and suppresses the Wnt/beta-catenin signal via ISGylation of beta-catenin. Gut 59, 907-917 (2010).

20. Lee, J. H., Park, M. S. \& Chung, I. J. Induction of 90K-specific Cytotoxic T Lymphocytes for Colon Cancer Immunotherapy. Immune Netw. 10, 206-211 (2010).

21. Piccolo, E. et al. Prognostic relevance of LGALS3BP in human colorectal carcinoma. J. Transl. Med. 13, 248 (2015).

22. Zambelli, D. et al. Biological indicators of prognosis in Ewing's sarcoma: an emerging role for lectin galactoside-binding soluble 3 binding protein (LGALS3BP). Int J. Cancer 126, 41-52 (2010).

23. Ma, N., Liu, Q., Hou, L., Wang, Y. \& Liu, Z. MDSCs are involved in the protumorigenic potentials of GM-CSF in colitis-associated cancer. Int J. Immunopathol. Pharmacol. 30, 152-162 (2017).

24. Li, W. et al. G-CSF is a key modulator of MDSC and could be a potential therapeutic target in colitis-associated colorectal cancers. Protein Cell. 7, 130-140 (2016).

25. Wang, T. et al. Galectin-3 contributes to cisplatin-induced myeloid derived suppressor cells (MDSCs) recruitment in Lewis lung cancer-bearing mice. Mol. Biol. Rep. 41, 4069-4076 (2014)

26. Udden, S. M. N. et al. NOD2 Suppresses Colorectal Tumorigenesis via Downregulation of the TLR Pathways. Cell Rep. 19, 2756-2770 (2017).

27. Zhao, $H$. et al. Cancer testis antigen 55 deficiency attenuates colitis-associated colorectal cancer by inhibiting NF-kappaB signaling. Cell Death Dis. 10, 304 (2019).

28. Xu, Y. L., Ding, C. L., Qian, C. L., Qi, Z. T. \& Wang, W. Retinoid acid induced 16 deficiency aggravates colitis and colitis-associated tumorigenesis in mice. Cell Death Dis. 10, 958 (2019).

29. Kishimoto, K. Matsumoto, K. \& Ninomiya-Tsuji, J. TAK1 mitogen-activated protein kinase kinase kinase is activated by autophosphorylation within its activation loop. J. Biol. Chem. 275, 7359-7364 (2000). 
30. Liu, Z. et al. Activation of TGF-beta activated kinase 1 promotes colon mucosal pathogenesis in inflammatory bowel disease. Physiol. Rep. 5, e13181 (2017).

31. West, N. R., McCuaig, S., Franchini, F. \& Powrie, F. Emerging cytokine networks in colorectal cancer. Nat. Rev. Immunol. 15, 615-629 (2015).

32. Fukata, M. et al. Toll-like receptor-4 promotes the development of colitisassociated colorectal tumors. Gastroenterology 133, 1869-1881 (2007).

33. Noma, N. et al. Involvement of NF-kappaB-mediated expression of galectin-3binding protein in TNF-alpha-induced breast cancer cell adhesion. Oncol. Rep. 27, 2080-2084 (2012).

34. Steinbach, E. C. \& Plevy, S. E. The role of macrophages and dendritic cells in the initiation of inflammation in IBD. Inflamm. Bowel Dis. 20, 166-175 (2014).

35. Li, J. et al. TAK1 inhibition attenuates both inflammation and fibrosis in experimental pneumoconiosis. Cell Discov. 3, 17023 (2017).

36. Kim, S. I. \& Choi, M. E. TGF-beta-activated kinase-1: new insights into the mechanism of TGF-beta signaling and kidney disease. Kidney Res. Clin. Pract. 31, 94-105 (2012)

37. Singh, A. et al. TAK1 inhibition promotes apoptosis in KRAS-dependent colon cancers. Cell 148, 639-650 (2012).

38. Totzke, J. et al. Takinib, a Selective TAK1 Inhibitor, Broadens the Therapeutic Efficacy of TNF-alpha Inhibition for Cancer and Autoimmune Disease. Cell. Chem. Biol. 24, 1029-1039 e1027 (2017).

39. Melisi, D. et al. Modulation of pancreatic cancer chemoresistance by inhibition of TAK1. J. Natl Cancer Inst. 103, 1190-1204 (2011).
40. Piro, G. et al. TAK1-regulated expression of BIRC3 predicts resistance to preoperative chemoradiotherapy in oesophageal adenocarcinoma patients. Br. J. Cancer 113, 878-885 (2015).

41. Cai, P. C. et al. Elevated TAK1 augments tumor growth and metastatic capacities of ovarian cancer cells through activation of NF-kappaB signaling. Oncotarget 5, 7549-7562 (2014).

42. Iriondo, O. et al. TAK1 mediates microenvironment-triggered autocrine signals and promotes triple-negative breast cancer lung metastasis. Nat. Commun. $\mathbf{9}$, 1994 (2018).

43. Gabrilovich, D. I. \& Nagaraj, S. Myeloid-derived suppressor cells as regulators of the immune system. Nat. Rev. Immunol. 9, 162-174 (2009).

44. Noguchi, M., Hiwatashi, N., Liu, Z. X. \& Toyota, T. Increased secretion of granulocyte-macrophage colony-stimulating factor in mucosal lesions of inflammatory bowel disease. Digestion 63, 32-36 (2001).

45. Cho, H. et al. Cancer-Stimulated CAFs Enhance Monocyte Differentiation and Protumoral TAM Activation via IL6 and GM-CSF Secretion. Clin. Cancer Res. 24, 5407-5421 (2018).

46. lacovazzi, P. A. et al. Are 90K/MAC-2BP serum levels correlated with poor prognosis in HCC patients? Preliminary results. Int J. Biol. Markers 18, 222-226 (2003).

47. Marchetti, A. et al. Expression of 90K (Mac-2 BP) correlates with distant metastasis and predicts survival in stage I non-small cell lung cancer patients. Cancer Res. 62, 2535-2539 (2002). 